\title{
Neutrophil Count Decreased, CTCAE
}

National Cancer Institute

\section{Source}

National Cancer Institute. Neutrophil Count Decreased, CT CAE. NCI Thesaurus. Code C143704.

A finding based on laboratory test results that indicate a decrease in number of neutrophils in a blood specimen. 